\title{
Effects Of Rebar Tying Machine On Trunk Flexion And Productivity
}

\author{
Peter Vi, Hon.BSc., M.Eng \\ Construction Safety Association of Ontario (Toronto, Canada)
}

\begin{abstract}
A before-and-after experimental design was conducted to evaluate the potential reduction in the risk of musculoskeletal injuries to concrete reinforcement workers when using an automatic rebar tying machine. Eleven (11) concrete reinforcement workers participated in this experiment. All dependent variables (trunk posture, and rebar tying time) were measured before and after implementing the rebar tying machine. The results of the study indicated that working with a rebar tying machine significantly reduced the magnitude and duration of exposure to awkward trunk posture. Tying time was reduced when participants used the machine. Based on trunk posture exposure and rebar tying time, it is concluded that the rebar tying machine can be an effective tool to reduce the frequency and duration of severe trunk flexion and increase productivity among concrete reinforcement workers.
\end{abstract}

\section{Introduction}

The risk of injury to concrete reinforcement workers is well documented. According to a recent study conducted by the Washington State Department of Labor and Industries, rodworkers have the highest rate (3,997 per 10,000 FTEs/Year) of non-traumatic soft tissue injuries when compared to 300 industrial occupation classes (Silverstein and Kalat, 1999). Similar findings are also evident in Ontario, where rodworkers have a higher lost-time injury (LTI) rate due to non-traumatic musculoskeletal injury than the construction average. The proportion of nontraumatic musculoskeletal injuries of the back and upper limb body parts was also found to be higher than the construction trades average between the years 1996 and 2002.

In a study of the association between occupational factors, other determinants, and the occurrence of sciatic pain, Riihimaki et al (1989) found that rodworkers experienced an increase in the risk of sciatic pain in both a cross-sectional and a prospective study. In similar research, Wickstrom et al (1985) and Riihimaki (1985) found that reported minor back accidents (i.e. an event that differed from the normal course of work and caused sudden, unexpected strain on the musculoskeletal system) were more than ten times as common in reinforcing work than in painting. Injuries to the musculoskeletal system, as registered by insurance companies, were also over three times more common in reinforcing work than in painting. Based on these findings, Riihimaki et al (1989), Riihimaki (1985), and Wickstrom et al (1985) concluded that the heavy work of concrete reinforcement increases the risk of musculoskeletal disorders when compared with the less burdensome work of house painting. Awkward postures, manual material handling, and back accidents seem to be an important risk factor for the occurrence of back pain.

Dababneh and Waters (2000) reviewed the literature on the ergonomics issue of rebar tying, and concluded that properly designed powered tying tools may be the best ergonomic solution. Extended tool-body design or extended arm attachments can be used to eliminate the need for stooping when working on a horizontal platform (see Figure 1). Also, tools with a pistol grip would allow workers to tie horizontal or vertical rebar while keeping their wrist straight and reducing the need to perform high twisting or bending motion of the wrists (Vi, 2003).

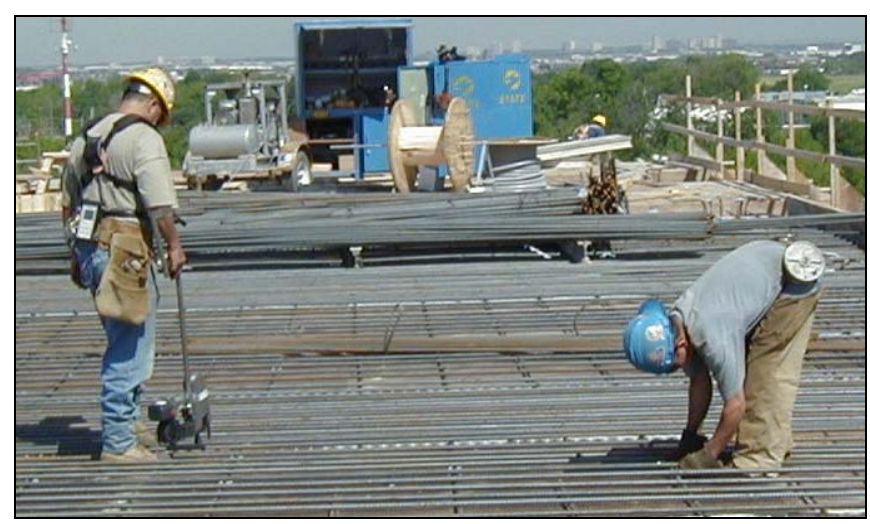

Figure 1: Rebar tying using a rebar tying machine (left) and manual tying using pliers (right). 
Although there are many potential benefits in using the rebar tying machines, their effectiveness in a field setting has never been verified. The objective of this study was to conduct an intervention study in the field setting to verify the potential reduction in risk of musculoskeletal disorders when using a rebar tying machine with arm extension attachment as an alternative work method. A secondary objective was to evaluate the productivity differences between manual tying with pliers and using the rebar tying machine.

\section{Method}

\section{Participants:}

Eleven (11) rodworkers participated in this experiment. All participants were unionized workers from the International Association of Bridge, Structural, Ornamental, and Reinforcing Ironworkers, Local 721. They were of average stature, $170 \mathrm{~cm}(+$ $7.9 \mathrm{~cm})$, body weight, $84.5 \mathrm{~kg}(+11.2 \mathrm{~kg})$, and age, 47 years $(+12$ year). The participants had no medical problems, and each signed an informed consent form.

\section{Study Design:}

A before-and-after design approach was used in this study to evaluate the effectiveness of the rebar tying machine with an extension arm attachment. Only ground level rebar production was used to evaluate the intervention. Before implementing the intervention, rebar tying time and trunk work postures while performing manual tying were quantified. After the initial observation, each participant was trained and given the rebar tying machine for use in normal ground level rebar production. Training covered proper use of the machine, including procedures to change the spool wire, trouble shooting, and machine maintenance. Each participant was allowed to use the rebar tying machine for three months. After the intervention period, rebar tying time and trunk work postures while performing rebar production with the tying machine were quantified. The repeated measurement of all dependent variables (i.e., before and after) allowed comparison of the differences between the two work methods.

\section{Dependent Variables:}

To investigate the potential benefits and reduced risks of musculoskeletal injury, rebar tying tasks were evaluated, using real-time continuous trunk posture exposure, and rebar tying time. All dependent variables were obtained from actual job condition. For each participant, continuous observation was made across two days. For each day of observation however, the data collection period was limited to four hours.

Once the data collection instruments were setup, workers were instructed to perform all the regular duties within ground level rebar production. This includes material handing of rebar steel and tying rebar. The procedures for collecting all dependent variables were repeated similarly before and after the intervention. Detailed methods of collecting the dependent variables are outlined in the following section.

\section{Gyroscope:}

The MicroStrain 3DM-G (Vermont, USA) gyroscope was used in this study to quantify realtime continuous trunk postures. The $3 D M-G$ is a self-contained sensor system that measures the three degrees of its orientation in space with respect to Earth's cardinal axes (i.e., the Z-axis pointing down through the center of the Earth, the $\mathrm{X}$-axis pointing north and the $\mathrm{Y}$-axis pointing east). The measurements by the 3DM-G include pitch, roll and yaw.

The 3DM-G was attached to a Biometric DataLog II system (Biometrics Ltd, Gwent, UK) for data collection and storing. To measure the trunk posture, the 3DM-G was attached on the midsection of the posterior scapula (Figure 2). In this study, only the roll (flexion/extension of the trunk) orientation was measured. The $3 D M-G$ was sampled at $100 \mathrm{~Hz}$ and low-pass filter at $4 \mathrm{~Hz}$ to reduce the high frequency noise. Trunk posture was continuously measured on all participants for duration of between two and four hours over two consecutive days. Awkward trunk posture exposure was analyzed using the amplitude probability distribution function (APDF) technique proposed by Jonsson (1982). Two exposure levels (i.e., median and peak levels) were obtained from the $50^{\text {th }}$ and $90^{\text {th }}$ percentiles of the distribution function according to Jonsson's definitions (1982). These were used to analyze the differences in exposure levels between the two work methods. Exposure variation analysis (EVA) proposed by Mathiassen and Winkel (1991) was also used to evaluate the differences in the magnitude, duration, and the distribution of the patterns of trunk posture exposure between the two rebar tying methods. 


\section{Rebar tying time:}

Rebar tying time was measured in this study to evaluate the differences in productivity between the two tying methods. Measurements of tying time for manual tying and machine tying were quantified from videotapes of ground level rebar production. For each rodworker, approximately two hours of continuous video analyses of each tying method were conducted. The number of rebar ties within the period of observation was expressed as the time (in seconds) required to perform a rebar tie (i.e., seconds per tie).

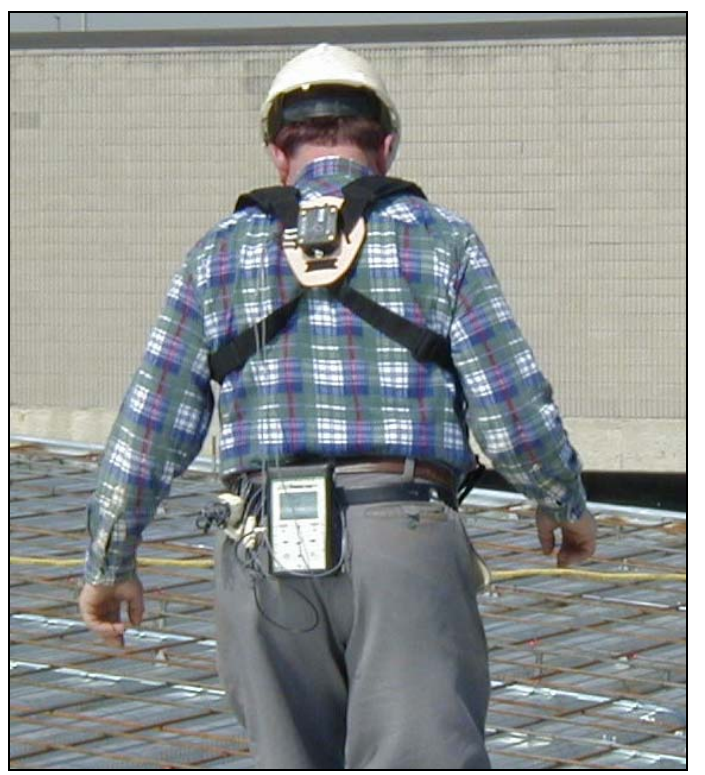

Figure 2: Rear view of the Gyroscope and DataLog II used to collect trunk posture.

\section{Data Analysis:}

SPSS (version 10) was used to analyze the collected data. Statistical tests with $\mathrm{p}<0.05$ were considered significant. Univariate analysis using Paired T-test and Wilcoxon nonparametric statistics were used to determine the differences between traditional versus alternative methods.

\section{Results}

\section{Trunk Posture}

A contour graph detailing the exposure variation analyses of the trunk posture for manual tying and machine tying is shown in Figure 3 and 4 . The contour graph is a 3-dimensional view illustrating the magnitude, frequency, and duration of trunk posture simultaneously during ground-level rebar work. Each tying method was separated into two different graphs for comparison. As shown in Figure 3, during manual tying with pliers, the highest level of trunk postures was heavily skewed to the far left and right (" $U$ " shape), indicating that manual tying with pliers consisted of long periods of neutral postures $\left(<20^{\circ}\right)$ and very extreme awkward trunk postures $\left(>60^{\circ}\right)$. During machine tying however, the distribution of the magnitude, frequency, and duration of the trunk posture was found to be concentrated between the trunk angles of greater than $-10^{\circ}$ and less than $50^{\circ}$ (see Figure 4).

A summary of the overall average trunk posture amplitude probability distribution function (APDF) for each tying method across all participants is shown in Figure 5. The amplitude of the median and peak trunk posture level was found significantly $(\mathrm{p}<0.05)$ higher when participants tied rebar with pliers as compared to tying machine.

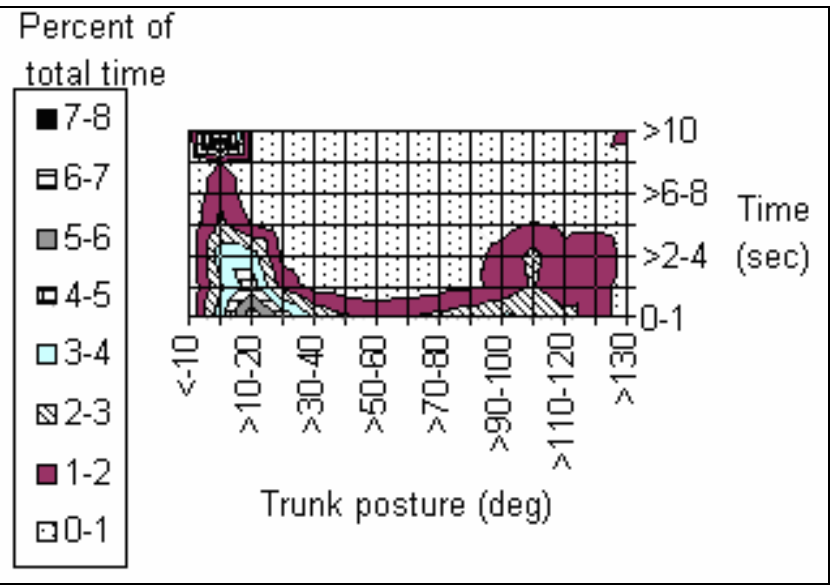

Figure 3: A contour graph detailing trunk posture while tying with pliers.

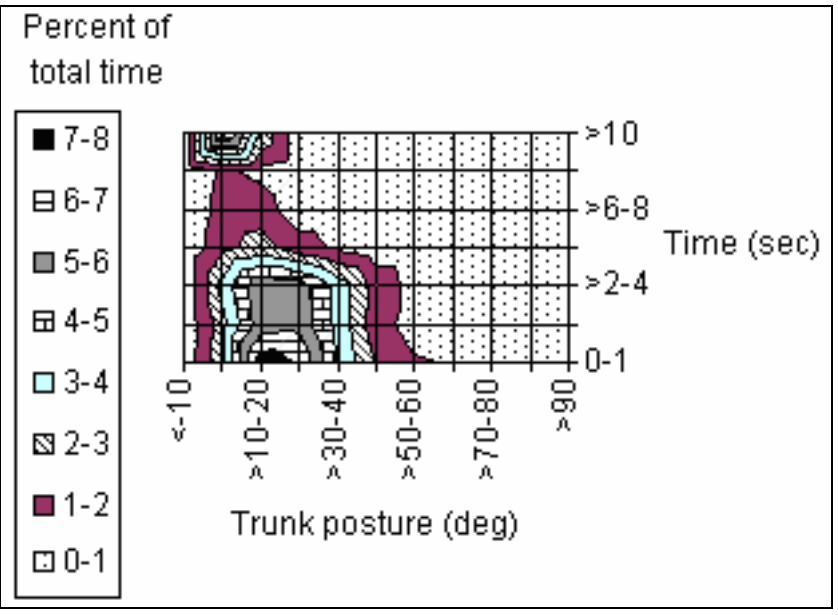

Figure 4: A contour graph detailing trunk posture while using the rebar tying machine. 


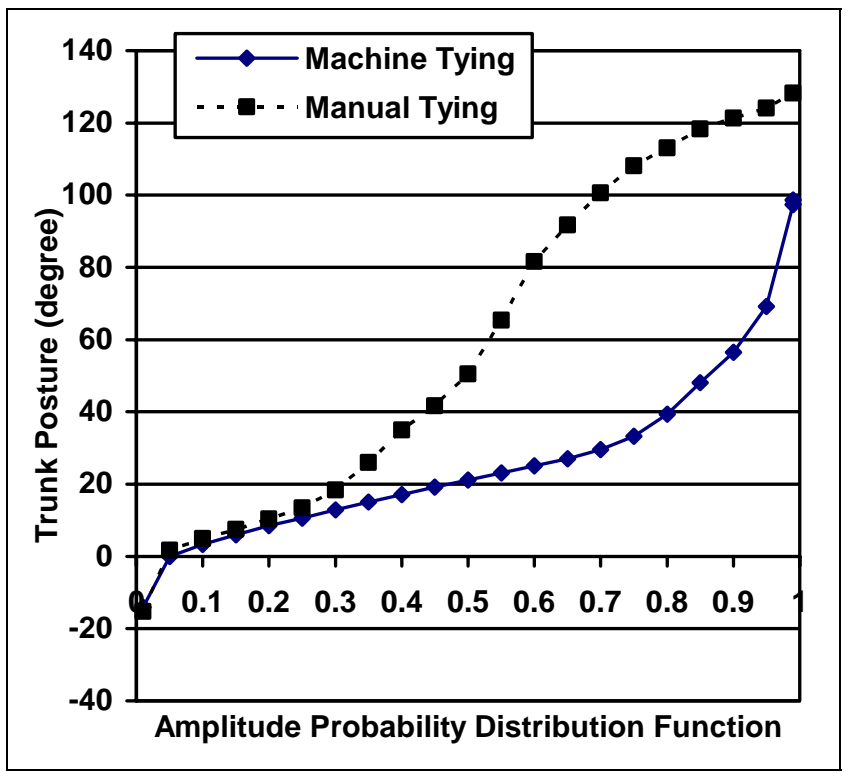

Figure 5: Average APDF of the trunk posture.

\section{Rebar Tying Time}

Paired t-test statistics revealed that there was a significant difference $(\mathrm{p}<0.05)$ in the rebar tying cycle time between the two work methods (see Figure 6). Using the traditional manual method the participants finished with an average rebar tying cycle time of 8.9 seconds. Using the rebar tying machine, the participants finished with an average rebar tying cycle time of 4.2 seconds - a decrease of 52\% in comparison to the traditional method.

\section{Discussion}

\section{Trunk Posture Exposure}

In a controlled-experimental study, Vi (2003) found significant $(<0.01)$ differences in wrist and arm motions (flexion/extension, radial/ulnar, and pronation/supination) between the rebar tying machine and manual tying methods. For all planes of motion, the rebar tying machine exposed the participants to significantly lower wrist activities (i.e., acceleration of the wrist) than when tying task with pliers. Low-back compression forces were also indirectly measured from electromyography. Significantly $(\mathrm{p}<0.05)$ lower low-back compression forces were found when workers used the rebar tying machine.

In the present study, significant differences in the magnitude, frequency and pattern of the trunk posture were also observed between the two tying methods.
EVA method demonstrated that rodworkers using pliers (manual method) spent a significantly higher amount of their work time in larger trunk angles in combination with longer time periods than rodworkers using the rebar tying machine. The pattern of exposure revealed a " $U$ " shape relationship between trunk posture and the continuous time period of the exposure level (see Figure 3). EVA and APDF methods also showed that the pattern of the trunk posture in manual tying with pliers differs most strongly for work time in trunk flexion greater than $45^{\circ}$. With the rebar tying machine, however, the rodworkers' trunk posture was mostly confined between the trunk angles of greater than $-10^{\circ}$ and less than $40^{\circ}$ (see Figure 4).

Long-term exposure to awkward trunk posture while tying rebar can expose workers to a high risk of lowpain back. Evidence from past research indicates the strong association between exposure to awkward trunk posture and risk of low-back pain (LBP). A large case-control study conducted by Norman et al (1998) found a positive relationship between peak trunk flexion and reporting of LBP. The odds ratio (OR) for the risk of LBP between the case and control based on the peak trunk flexion variable was 2.4 (95\% CI 1.5-3.8). Similarly Marras et al (1995) observed an OR of 1.6 (95\% CI 1.31-1.93). The first evidence of a positive relationship between the percentages of time exposed to non-neutral trunk flexion ( $>20^{\circ}$ flexion) and risk of LBP was found by Punnett et al (1991). In the Punnett et al (1991) study, multivariate analyses that adjusted for covariates demonstrated that time in non-neutral postures was strongly associated with back disorders (OR 8.09, 95\% CI 1.4-44).

\section{Productivity}

As measured by tying time, productivity was very much dependent on the type of tying method. In this study, the rebar tying machine was found to be superior to traditional manual tying. On average, a $52 \%$ decrease in the time to tie one rebar was found when using the rebar tying machine. This increase in productivity is positive because it allows workers to be more productive without increasing the risk of musculoskeletal disorders to the back and upper extremities. 


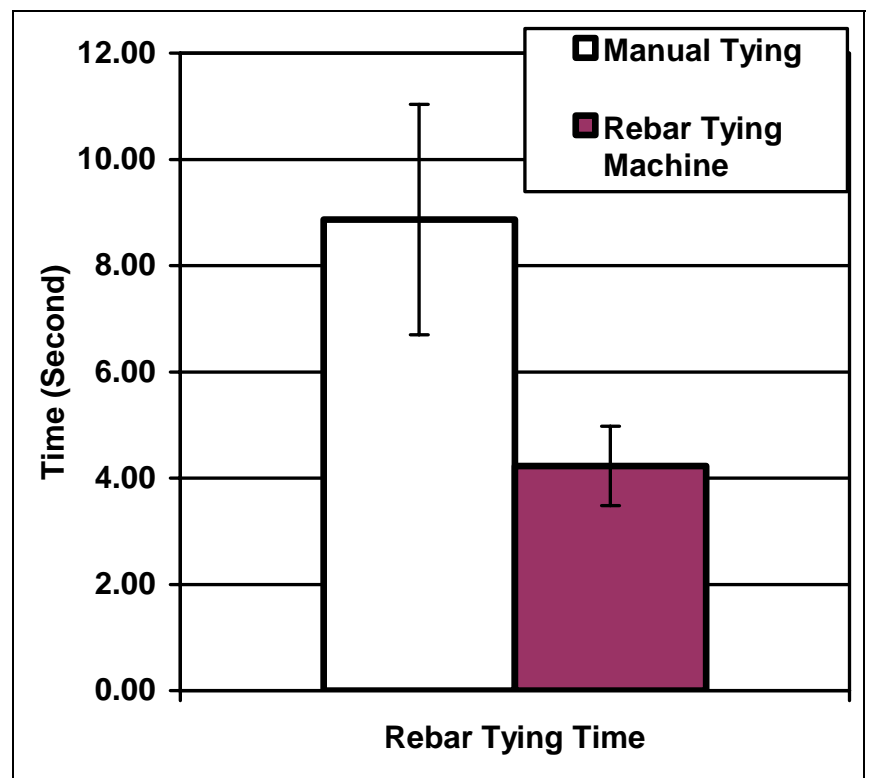

Figure 6: Rebar tying cycle time between the two tying methods. A cycle time is defined as the time required to tie one connection.

\section{Reference}

Dababneh A., and Waters T., (2000). Ergonomics of rebar tying. Applied Occupational and Environmental Hygiene, 15(10): 721-727.

Holmstrom E., (1992). Musculoskeletal disorders in construction workers: Related to physical, psychosocial, and individual factors. Department of physical therapy, Lund University, Lund, Sweden.

Jonsson B., (1982). Measurement and evaluation of local muscular strain on the shoulder during constrained work. Journal of Human Ergology 11:7388.

Norman R., Wells R., Neumann P., et al., (1998). A comparison of peak vs cumulative physical work exposure risk factors for the reporting of low back pain in the automotive industry. Clinical Biomechanics, 13: 561-573.

Marras W., Lavender S., Leurgans S., et al., (1995). Biomechanical risk factors for occupationally related low back disorders. Ergonomics, 38(2): 377-410.

Punnett L., Fine L., Keyserling M., Herrin G., and Chaffin D., (1991). Back disorders and nonneutral trunk postures of automobile assembly workers. Scandinavian Journal of Environmental Health, 17:337-346.
Robson L., Shannon H., Goldenhar L., and Hale A., (2001). A guide to evaluating the effectiveness of strategies for preventing work injuries: How to show whether a safety intervention really works. Department of health and human services, NIOSH publication No. 2001-119.

Silverstein B., and Kalat J., (1999). Non-traumatic soft tissue MSDs, 1990-1997. Safety \& Health Assessment and Research for Prevention (SHARP) Program, Report \#40-3-99.

Vi P., (2003). Reducing risk of musculoskeletal disorders through the use of rebar-tying machines. Applied Occupational and Environmental Hygiene, 18: 649-654.

\section{ACKNOWLEDGMENTS}

This project was funded by a research grant provided by the Workplace Safety and Insurance Board (Ontario). The author would also like to acknowledge the International Association of Bridges Structural and Ornamental Iron Workers Local 721 for providing access to the study site and work materials. The CSAO Rodworkers Labour-Management Health \& Safety Committees for their advice and support. 